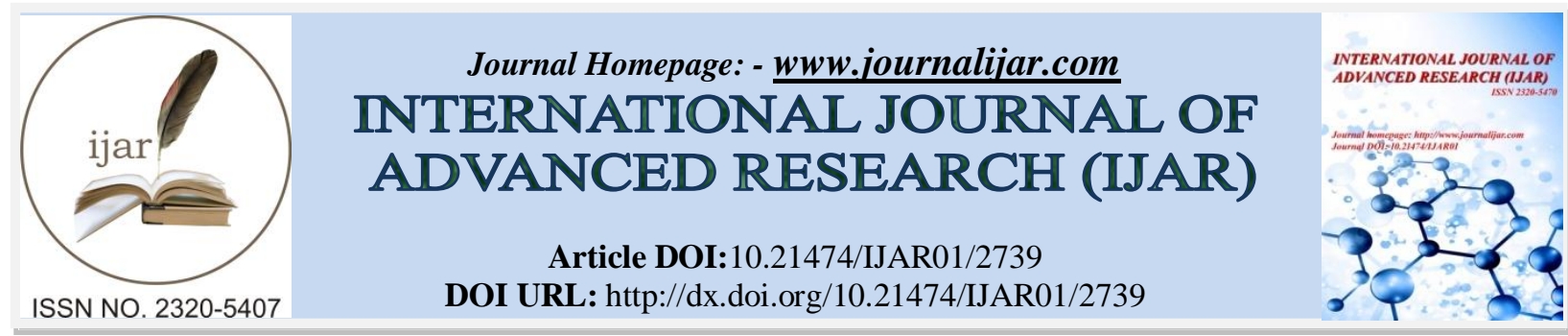

RESEARCH ARTICLE

\title{
LAPAROSCOPY ROLE IN DIAGNOSIS AND MANAGEMENT OF UNEXPLAINED/NONSPECIFIC ABDOMINAL PAIN.
}

\author{
Faisal Abdullah Al Qahtani ${ }^{1}$, Hamad Mohammed Al Selaimy ${ }^{2, *}$ and Saad Mohammed Al Muqrin ${ }^{(2)}$ \\ 1. Medical Intern, College of Medicine, King Saud University, Riyadh, Saudi Arabia. \\ 2. College of Medicine, King Saud bin Abdulaziz University for Health Sciences, Riyadh, Saudi Arabia.
}

\section{Manuscript Info}

………………….

Manuscript History

Received: 15 November 2016

Final Accepted: 17 December 2016

Published: January 2017

Key words:-

nonspecific abdominal pain,

laparoscopy, abdomen, diagnosis

\section{Abstract}

Background:unexplained/nonspecific abdominal pain makes up a high percentage of surgical admissions to hospitals, both in emergency department, and as elective admissions, with a substantial diagnostic dilemma.

Objective: The aim of this study was to determine if laparoscopy has any significant role in the diagnosis and management of unexplained/nonspecific abdominal pain.

PatientsandMethods: All patients who presented to the hospital with abdominal pain with no identifiable etiology and after clinical examination, assessment and investigations were labeled as unexplained/nonspecific abdominal pain, and after that underwent diagnostic laparoscopyto reach a definitive diagnosis were included in the study.

Results: 127 patients were included in the study. Females accounted for $67 \%(\mathrm{n}=85)$ and males $33 \%(\mathrm{n}=42)$. The mean age was 28 years (ranged from18-58 years).Out-patient department was the commonest mode of admission,99 (78.4\%) patients. Patients who presented with unexplained/nonspecific abdominal pain in the lower part of abdomen accounted for $29 \%(\mathrm{n}=37)$, followed by $25 \%(\mathrm{n}=32)$ with right lower abdominal pain, and $22 \%(\mathrm{n}=28)$ with periumbilical pain radiating to right lower abdomen. Diagnosis was established in $85 \%(n=108)$ of patients. In $15 \%(\mathrm{n}=19)$ no pathology was found. The most common diagnosis was pathology of appendix in $33 \%(n=42)$ of patients followed by pelvic pathology in $20 \%(\mathrm{n}=25)$, and abdominal tuberculosis in $16 \%(\mathrm{n}=20)$ of patients. Most of the patients $(42 \%$, $\mathrm{n}=53$ ) stayed in the hospital for $24 \mathrm{~h}$. There were no major postoperative complications and no readmissions.

Conclusion: Laparoscopy has a definitive role in the diagnostic dilemma associated with unexplained/nonspecific abdominal pain. It has at the same time a role in treatment, decreasing mortality, morbidity, cost, hospital stay, and improving quality of life relative to observation; hencelaparoscopy is an effective diagnostic and therapeutic modality in the management of patients with unexplained/nonspecific abdominal pain. 


\section{Introduction:-}

Abdominal pain is a common complaint both in general practice and in hospitals, with which patients present to emergency department. ${ }^{(1)}$ Nonspecific abdominal pain (NSAP), is generally defined as acute abdominal pain of under 7 days' duration, and for which there is no diagnosis after examination and baseline investigations, is a common cause of emergency surgical hospitalization. ${ }^{(1)}$ Nearly $25 \%$ of patients have nonspecific abdominal pain. ${ }^{(2)}$ More than $38 \%$ of the patients with chronic abdominal pain have no specific etiological diagnosis made at the end of the diagnostic workup. (3), (4) Many organic and functional diseases can cause abdominal pain. The most common organic conditions include intestinal adhesions, (5), (6) biliary causes, (7), (8) and appendicular causes, (9) while functional conditions include irritable bowel disease, (10) functional dyspepsia, (11) and various motility disorders.Many of patients have ongoing symptoms and cannot be discharged, undergo multiple, often expensive, unneeded investigations and have repeated readmissions. A lot of cases of NSAP are mimicking acute or chronic appendicitis. Most patients with NSAP are referred as possible cases of acute appendicitis, frequently withperiumbilical pain and tenderness that radiates to right iliac fossa (RIF). A number of these may erroneously undergo operation for suspected appendicitis, some are put on anti-tuberculosis therapy, while females mostly end up taking anti-androgens. ${ }^{(12)}$ One study showed that, NSAP was diagnosed in $30 \%$ of 135 patients who are undergoing appendicectomy operations. ${ }^{(1)}$ Patients may undergo unnecessary appendectomy operations and may reiterate with symptoms even after surgery. A definite diagnosis is not always achievable, however, with noninvasive imaging modalities including contrast radiology, computed tomography (CT), magnetic resonance imaging (MRI), and ultrasonography. ${ }^{(13)}$ In addition, minimally invasive procedures like paracentesis, endoscopy fail to reach a diagnosis as well at times.Laparoscopy is a minimally invasive surgical procedure in which the peritoneal cavity and intrabdominal organs can be visualized in order to detect pathology. It has both a diagnostic as well as a therapeutic potential and it is especially useful in patients with equivocal signs and those who are hemodynamically stable not requiring urgent surgical intervention. It is a safe and effective tool and can establish the etiology and allows for appropriate interventions in such cases. ${ }^{(14)}$ After giving symptomatic treatment to patients, normal diagnostic laparoscopy may allow the surgeon to discharge patients early from hospital. This study was conducted to highlight the role of laparoscopy in unexplained/nonspecific abdominal pain.

\section{Materials and Methods:-}

This study was conducted out in Department of Surgery, King Khalid University Hospital (KKUH), Riyadh, Saudi Arabia for a period of 3 years, from July 2011 to June 2014. All patients of either sex and age, who were admitted through emergency department or as an elective through outpatient department and presented with acute or chronic nonspecific abdominal pain which was of unclear etiology, in whom medical history, clinical assessment, routine laboratory investigations and radiological evaluations failed to make a definite diagnosis, were included. After acquiring detailed history and clinical examination, relevant blood investigations taken, X-ray abdomen, ultrasound, and CT scan were performed when indicated. Laparoscopy was performed, after completion of all the necessary hematological, biochemical, radiological, and ascetic fluid analysis, endoscopic evaluation, imaging techniques, and Tuberculin sensitivity test, in any chronic abdominal condition in which the etiology was unknown.Depending on the underlying pathology, therapeutic intervention was performed with either open laparotomy or laparoscopic technique.A form was used to keep a record of all the patients' data in terms of medical history, clinical examination, laboratory investigations and laparoscopic findings. The time for hospital stay was defined as time from moment of admission until patient discharge.A single antibiotic for prophylaxis was given preoperatively.Patients informed-consent form approved by our Institution's Ethics Committee was taken before operation. Also, patients were informed about the possibility of conversion of laparoscopic surgery to an open procedure depending on preoperative findings. Pneumoperitoneum was established using veress needle in periumbilical region. After establishment of the pneumoperitoneum, a standard three-trocar technique was used (10$\mathrm{mm}$ optic via periumbilical trocar and two 5-mm lateral trocars), followed by insertion of additional ports where therapeutic intervention was required.The findings and results of laparoscopy were recorded. Biopsy specimens were sent for histopathology to confirm the diagnosis. Outcome measures included diagnosis made, duration of surgery, duration of hospital stay and postoperative complications. Data were analyzed using Statistical Package for the Social Sciences Version 20.0 (SPSS Inc., Chicago, IL, USA) and Microsoft Excel 2010. Categorical data was presented as frequency and percentage, and numerical data as means and standard deviations. 


\section{Results:-}

The common mode of admission to hospital was through outpatient department ( $n=99,78.4 \%)$. The common clinical presentations were as follow: nonspecific abdominal pain in the lower part abdomen in $29 \%(\mathrm{n}=37)$ patients, with right lower abdominal pain in $25 \%(\mathrm{n}=32)$ and $22 \%(\mathrm{n}=28)$ with periumbilical pain radiating to right lower abdomen.(detailed inTable 1).

Table 1:-Clinical presentations

\begin{tabular}{|l|l|l|}
\hline Site of abdominal pain & Number of patients (N) & Percentage (\%) \\
\hline $\begin{array}{l}\text { Pain in lower abdomen } \\
\text { (RIF, hypogastrium, LIF) }\end{array}$ & $\mathbf{3 6}$ & $\mathbf{2 8 . 4}$ \\
\hline Pain in RIF periumbilical/central & $\mathbf{3 0}$ & $\mathbf{2 4}$ \\
\hline $\begin{array}{l}\text { Pain in } \\
\text { abdomen }\end{array}$ & $\mathbf{2 2}$ \\
\hline Pain in hypogastrium & $\mathbf{9}$ & $\mathbf{6 . 8}$ \\
\hline Pain in LIF & $\mathbf{9}$ & $\mathbf{6 . 8}$ \\
\hline Pain in right lumbar region & $\mathbf{7}$ & $\mathbf{5 . 6}$ \\
\hline Pain in RIF and hypogastrium & $\mathbf{4}$ & $\mathbf{3 . 4}$ \\
\hline Pain in left lumbar region & $\mathbf{3}$ & $\mathbf{2 . 5}$ \\
\hline Total & $\mathbf{1 2 7}$ & $\mathbf{1 0 0}$ \\
\hline
\end{tabular}

In 43\% ( $\mathrm{n}=55)$ of patient's abdominal ultrasound was normal. A common finding that was noted on abdominal and pelvic ultrasound, was distended bowel loops in right iliac fossa. Benign prostatic hypertrophy was reported in three patients. Pelvic ultrasound in 81 of 85 females was normal. In the remaining patients, minimal free fluid in cul-desac was reported. All subjects underwent CT scanning, out of which, 52.5\% $(\mathrm{n}=66)$ patients had a change in findings when compared with the findings on ultrasonography.The CT scan was better able to show retroperitoneal/mesenteric lymphadenopathy and dilatation of gut loops. Thirty-four subjects out of 127 cases (27.2\%) had altogether new findings, while $92(72.7 \%)$ cases had findings similar to the radiological means. Thirtysix out of these 64 had new findings along with the previous findings. Therefore, 70 out of the 127 subjects had new findings. After diagnostic laparoscopy, tissue diagnosis was achieved in 108 of the 127 subjects (85\%).Laparoscopic results showed inflamed appendix, appendicular fecaliths, mesenteric lymphadenitis,enlarged mesenteric lymph nodes, salpingitis, omentum at deep inguinal ring, adhesions in peritoneal cavity and pelvis, fluid in cul-de-sac and ovarian cysts, diverticulitis(as indicated in Table 2).

Table 2:-Laparoscopic findings

\begin{tabular}{|l|l|l|}
\hline Laparoscopic findings & Number of patients (N) & Percentage (\%) \\
\hline Inflamed appendix & $\mathbf{2 2}$ & $\mathbf{1 7}$ \\
\hline $\begin{array}{l}\text { Adhesions/bands } \\
\text { (postoperative/cong.) }\end{array}$ & $\mathbf{1 6}$ & $\mathbf{1 2 . 5}$ \\
\hline Appendicular faecoliths & $\mathbf{1 2}$ & $\mathbf{1 0 . 2}$ \\
\hline Enlarged mesenteric lymph nodes & $\mathbf{1 2}$ & $\mathbf{1 0}$ \\
\hline Salpingitis with fluid in cul-de-sac & $\mathbf{1 0}$ & $\mathbf{7 . 5}$ \\
\hline Ovarian cysts & $\mathbf{9}$ & $\mathbf{6 . 8}$ \\
\hline $\begin{array}{l}\text { Caesating mesenteric nodes with } \\
\text { ileal adhesions }\end{array}$ & $\mathbf{9}$ & $\mathbf{6 . 8}$ \\
\hline Fibrous/kinked appendix & $\mathbf{7}$ & $\mathbf{5 . 6}$ \\
\hline $\begin{array}{l}\text { Strictured terminal ileum with } \\
\text { lymph nodes }\end{array}$ & $\mathbf{4}$ & $\mathbf{3 . 4}$ \\
\hline Omentum/viscus in deep ring & $\mathbf{4}$ & $\mathbf{3}$ \\
\hline Meckel's diverticulitis & $\mathbf{1}$ & $\mathbf{1 . 1}$ \\
\hline Fimbrial cyst & $\mathbf{1}$ & $\mathbf{1 . 1}$ \\
\hline Worm in appendix & $\mathbf{1}$ & $\mathbf{1 . 1}$ \\
\hline No pathology found & $\mathbf{1 9}$ & $\mathbf{1 5}$ \\
\hline Total & $\mathbf{1 2 7}$ & $\mathbf{1 0 0}$ \\
\hline
\end{tabular}


Final diagnosis was made in $108(85.2 \%)$ patients. In 19 patients (14.7\%) diagnosis could not be established. All laparoscopic findings were confirmed by histopathology. Inflamed appendix was the most common diagnosis made in $22(17 \%)$ patients with rest of diagnoses detailed in $\underline{\text { Table } 3}$.

Table 3:- Final diagnosis

\begin{tabular}{|l|l|l|}
\hline Final diagnosis & Number of patients (N) & Percentage (\%) \\
\hline Appendicular pathology & $\mathbf{4 2}$ & $\mathbf{3 3}$ \\
\hline Pelvic pathology & $\mathbf{2 6}$ & $\mathbf{2 0 . 4}$ \\
\hline Abdominal tuberculosis & $\mathbf{2 0}$ & $\mathbf{1 6}$ \\
\hline Mesentricadenitis & $\mathbf{6}$ & $\mathbf{4 . 5}$ \\
\hline Postoperative adhesions & $\mathbf{4}$ & $\mathbf{3 . 4}$ \\
\hline Early inguinal hernia & $\mathbf{4}$ & $\mathbf{3 . 4}$ \\
\hline Congenital bands & $\mathbf{3}$ & $\mathbf{2 . 2}$ \\
\hline Lymphoma & $\mathbf{1}$ & $\mathbf{1}$ \\
\hline Meckel's diverticulitis & $\mathbf{1}$ & $\mathbf{1}$ \\
\hline
\end{tabular}

The average length of the operative time was 58.7 minutes and the maximum duration was 96minutes. Among the postoperative complications $10(8 \%)$ patients developed wound inflammation/infection, $8(6.8 \%)$ had post-operative fever and chest infection, 6 (4.5\%) with post-operative fever alone. Follow-up was done weekly for 1 month, then seen once monthly for 3 months. No major complications were reported and seven patients came back with complaints of recurrent pain who were subjected to further investigations and imaging modalities like CT, MRI, MRCP (magnetic resonance cholangiopancreatography) toreach adefinite diagnosis.

\section{Discussion:-}

Unexplained/nonspecific abdominal pain still remains a diagnostic dilemma. In many cases, despite all the routine laboratory investigations and imaging modalities, a diagnosis could not be achieved. The challenge posed by patients with NSAP is extensive and noticeable. It accounts for an estimated 15-40\% of all emergency-department surgical admissions. ${ }^{(1)}$ The abdominal disease is complicated, and patients usually undergo a lot of costly investigations and even exploratory laparotomy for definite diagnosis. In such conditions, diagnostic laparoscopy is a better choice for the patient. It can directly give a high-field visualization of the abdominal cavity, provide adequate tissue sample for histopathological assessment, and in expert hands is an excellent therapeutic tool with cosmetic acceptable scars. When reviewing the literature, it reveals various outcomes of diagnostic laparoscopy in patients of undiagnosed abdominal pain to support its use in recurrent nonspecific abdominal pain. Some studies clearly support diagnostic laparoscopy role in such challenging situations and have shown a good accuracy in reaching a diagnosis in NSAPs. ${ }^{(15),(16)}$ Some other studies were not as supportive. ${ }^{(17)}$ In our study, the overall success was $88 \%$ which supports use of this diagnostic modality. A study done by Lippert et al.showed that diagnostic difficulties are more experienced in young females with lower abdominal pain and inconsistent features of appendicitis. ${ }^{(18)}$ It seems to be a better option to evaluate nonspecific lower abdominal pain in this gender class by diagnostic laparoscopy. This is similar to the study carried by $\mathrm{Ou}$ and Rowbotham in which diagnostic laparoscopy provided a definitive diagnosis in 76 of the 77 cases $(99 \%) .{ }^{(19)}$ In our study, more than half (85 out of 127) of patients were females. This strengthens the observation that nonspecific abdominal pain was common diagnostic problem in this group. Diagnostic laparoscopy provided a definitive diagnosis in $73(86.4 \%)$ out of 85 female patients in our study. In a similar study appendicular pathology was found in $75 \%$ cases, whereas in another study it was found in $40 \%$ patients. ${ }^{(20),(21)}$ These numbers were higher in our study in which appendicular pathology was found in $33 \%(n=42)$ of patients. In majority of cases in our study, the appendicular pathology was inflamed appendix with pelvic appendix as a common position, along with subserosal and reterocecal appendix. Females with findings of pelvic pathology and pelvic inflammatory disease (PID) were the second most common diagnosis reached with $26(20.4 \%)$, and abdominal tuberculosis was found in 20 (16\%) patients in a study which is similar to our results. ${ }^{(22)}$ Five male patients in the present study presented with a complaint of right groin pain that radiated to right iliac fossa. On diagnostic laparoscopy, findings were protrusion of omentum into deep inguinal ring in two patients and small bowel along with omentum in three others. Transabdominalpreperitoneal repair was done in all the five patients with no postoperative complications. Our results were in concordance to a study revealing similar results. ${ }^{(4)}$ Early laparoscopy also has the benefit that a number of therapeutic options were available. ${ }^{(23)}$ In this study, diagnostic laparoscopy has become therapeutic in many cases. Ovarian cysts could be drained and treated with immediate relief of symptoms. ${ }^{(24)}$ Purulent fluid collections secondary to pelvic inflammatory disease or diverticulitis could be drained. Early recognition of PID is crucial and enables early treatment that was important if 
complications such as infertility were to be minimized. Patients with an inflamed appendix can be dissected safely and effectively laparoscopically. Laparoscopic adhesiolysis was possible. ${ }^{(25)}$ In our study, there were some minor postoperative complications noted including wound inflammation/infection and post-operative fever, while the laparoscopy failed to make any diagnosis in 17 patients, which were put on follow-up for further assessment to reach a diagnosis. Seven were females (unmarried) of child bearing age, five were males $<12$ years of age, whereas the other females had a history of cesarean section with normal imaging studies. All of these patients attended the follow-up clinic for 8 weeks and then were being evaluated with other modalities during follow-up.

\section{Conclusion:-}

In conclusion, laparoscopy has an effective diagnostic role in evaluating patients with acute unexplained/nonspecific abdominal pain, in whom conventional methods of investigations have failed to reach a definitive cause. The therapeutic value of laparoscopy is also accepted and appreciated.

\section{References:-}

1. Sheridan WG, White AT, Havard T, Crosby DL. Non-specific abdominal pain: The resource implications. Ann R CollSurgEngl 1992; 74:181-5.

2. Kamin RA, Nowicki TA, Courtney DS, Powers RD. Pearls and pitfalls in the emergency department evaluation of abdominal pain. Emerg Med Clin North Am 2003; 21:61-72, vi.

3. Heafield R, Roe AM, Watkins R, Brodribb AJ, Brown C. Outcome of emergency surgical admissions for non-specific abdominal pain. Gut 1990;31: A1167.

4. El-Labban GM, Hokkam EN. The efficacy of laparoscopy in the diagnosis and management of chronic abdominal pain. J Minim Access Surg 2010; 6:95-9.

5. Peters AA, Van den Tillaart SA. The difficult patient in gastroenterology: Chronic pelvic pain, adhesions, and sub occlusive episodes. Best Pract Res ClinGastroenterol. 2007; 21:445-63.

6. Van Goor H. Consequences and complications of peritoneal adhesions. Colorectal Dis. 2007; 9:25-34.

7. Dumont RC, Caniano DA. Hypokinetic gallbladder disease: A cause of chronic abdominal pain in children and adolescents. J PediatrSurg. 1999; 34:858-61.

8. Johnson CD. ABC of the upper gastrointestinal tract. Upper abdominal pain: Gall bladder. BMJ. 2001; 323:1170-3.

9. Fayez JA, Toy NJ, Flanagan TM. The appendix as the cause of chronic lower abdominal pain. Am J ObstetGynecol. 1995; 172:122-3.

10. Mertz HR. Irritable bowel syndrome. N Engl J Med. 2003; 349:2136-46.

11. Tack J, Lee KJ. Pathophysiology and treatment of functional dyspepsia. J ClinGastroenterol. 2005;39: S211-6.

12. Galili O, Shaoul R, Mogilner J. Treatment of Chronic Recurrent Abdominal Pain: Laparoscopy or Hypnosis? J LaparoendoscAdvSurg Tech A. 2009; 19:93-6.

13. Cuesta MA, Borgstein PJ, Meijer S. Laparoscopy in the diagnosis and treatment of acute abdominal conditions. Clinical review. Eur J Surg 1993; 159:455-6.

14. nders RP, Mittendorf EA. Utility of laparoscopy in chronic abdominal pain. Surgery 2003; 134:549-52.

15. Domínguez LC, Sanabria A, Vega V, Osorio C. Early laparoscopy for the evaluation of nonspecific abdominal pain: A critical appraisal of the evidence. SurgEndosc 2011; 25:10-8.

16. Kapshitar' AV. The indications for performing diagnostic and therapeutic laparoscopy in emergency surgery on the abdominal organs. KlinKhir 1998;12-4.

17. Aslam MN, Ehsan O, Ali AA, Gondal KM, Choudhry AM. Diagnostic laparoscopic surgery: A good surgical tool. Pak J Surg 2001; 17:31-4.

18. Lippert V, Zaage J, Pilz F. Diagnostic laparoscopy and laparoscopic appendectomy in the diagnosis and therapy concept of abdominal pain of unknown origin. ZentralblChir 1998;123 Suppl 4:46-9.

19. Ou CS, Rowbotham R. Laparoscopic diagnosis and treatment of nontraumatic acute abdominal pain in women. J LaparoendoscAdvSurg Tech A 2000; 10:41-5.

20. Al-Bareeq R, Dayna KB. Diagnostic laparoscopy in acute abdominal pain: 5-year retrospective series. Bahrain Med Bull 2007; 29:1-5.

21. McCartan DP, Fleming FJ, Grace PA. The management of right iliac fossa pain-is timing everything? Surgeon 2010; 8:211-7.

22. Hossain J, al-Aska AK, al Mofleh I. Laparoscopy in tuberculous peritonitis. J R Soc Med 1992; 85:89-91.

23. Salky BA, Edye MB. The role of laparoscopy in the diagnosis and treatment of abdominal pain syndromes. SurgEndosc 1998; 12:911-4.

24. Gaitán H, Angel E, Sánchez J, Gómez I, Sánchez L, Agudelo C. Laparoscopic diagnosis of acute lower abdominal pain in women of reproductive age. Int J GynaecolObstet 2002; 76:149-58.

25. Poulin EC, Schlachta CM, Mamazza J. Early laparoscopy to help diagnose acute non-specific abdominal pain. Lancet 2000; 355:861-3. 